\section{SI ZUBIRI PUDIERA DISCUTIR CON LA BIOÉTICA ACTUAL: INTELIGENCIA Y NEUROCIENCIA}

\author{
Lydia Feito Grande \\ Universidad Complutense de Madrid \\ lydia.feito@med.ucm.es
}

\section{IF ZUBIRI COULD DISCUSS WITH CURRENT BIOETHICS: INTELLIGENCE AND NEUROSCIENCE}

Cómo citar este artículo/Citation: Feito Grande, L. (2016). Si Zubiri pudiera discutir con la bioética actual: inteligencia y neurociencia. Arbor, 192 (780): a330. doi: http://dx.doi. org/10.3989/arbor.2016.780n4006
Copyright: (c) 2016 CSIC. Este es un artículo de acceso abierto distribuido bajo los términos de la licencia Creative Commons Attribution (CC BY) España 3.0.
RESUMEN: Los filósofos son hijos de su tiempo. Sus enfoques, sus temas y sus abordajes son deudores de un momento histórico, de unos conocimientos y de un paradigma vigente a la altura de los tiempos en que les ha tocado vivir. Zubiri es buen ejemplo del filósofo que no quiere estar al margen de la ciencia y el conocimiento de su tiempo, pero no deja de ser un autor del siglo XX. La bioética actual expone una profusión de temas que, sin duda, hubieran fascinado a Zubiri, en tanto que atañen a la reflexión sobre la misma condición del ser humano. Específicamente, el desarrollo de las neurociencias propone un reto al pensamiento, que está generando el correlativo avance de la llamada Neuroética. Desde este nuevo escenario se aborda aquí un esbozo del diálogo que Zubiri podría haber planteado ante la neuroética del presente.

PALABRAS CLAVE: Zubiri; neuroética; bioética; neurociencia; conciencia; inteligencia; naturaleza; realidad.
ABSTRACT: Philosophers are products of their time. Their standpoints, themes and perspectives are a result of a historical time, a knowledge and a prevailing paradigm throughout their epoch. Zubiri is a good example of a philosopher who wants to be in touch with the science and knowledge of his time, but is an author from the XX century. Contemporary bioethics includes a profusion of themes that undoubtedly would have fascinated Zubiri, in as far as they relate to a reflection on the human condition itself. Specifically, the development of neurosciences is challenging to thought, and is producing a correlative development of so-called neuroethics. From this new scenario, this paper deals with those ideas that Zubiri would have discussed in the context of contemporary neuroethics.

KEYWORDS: Zubiri; neuroethics; bioethics; neuroscience; consciousness; intelligence; nature; reality. 


\section{INTRODUCCIÓN. LA BIOÉTICA ACTUAL COMO RETO INTELECTUAL: BIOÉTICA FUNDAMENTAL Y BIOÉTICA APLICADA}

Mi objetivo en esta intervención es mostrar brevemente algunos de los retos intelectuales que está generando la bioética actual, tanto en su dimensión más fundamental como en la más aplicada, específicamente en un terreno de notable expansión: la neuroética, es decir, el estudio de las implicaciones antropológicas, conceptuales y éticas de los resultados de las neurociencias, para plantear, más como interrogantes que como afirmaciones, cuáles son los puntos que a Zubiri le resultarían más interesantes y cuál sería su abordaje de los mismos.

Lamentablemente, Zubiri no tuvo la ocasión de asistir a los apasionantes descubrimientos de las últimas décadas. No pudo conocer cómo la biología, nueva reina de las ciencias, ha desbordado su campo en dos ámbitos específicos: la genética y la neurociencia. Sus impresionantes aportaciones han generado no pocos interrogantes filosóficos, desde el punto de vista de sus implicaciones para reflexiones antropológicas, como la que atañe a la existencia de una naturaleza humana, o éticas, como la que versa sobre la responsabilidad y la toma de decisiones. Estas dos ciencias han obligado a pensar que, dentro de la bioética, se pueden distinguir subdisciplinas como la GenÉtica y la Neuroética, cuyo objetivo es el análisis de todos esos interrogantes e implicaciones éticas que generan la genética y la neurociencia respectivamente.

La neuroética, y la bioética en general, tienen una dimensión fundamental y otra aplicada. La primera se refiere al estudio de los conceptos y teorías que puedan utilizarse en la interpretación de los datos, buscando las razones de su validez y aportando principios o sistemas explicativos desde los que pueda orientarse la segunda de las dimensiones, la que hace referencia a la aplicación de las teorías y principios a los problemas específicos de un campo, tratando de lograr un doble objetivo: encontrar solución a los conflictos y establecer, con ello, un modo de toma de decisiones acorde con un patrón de promoción de unos determinados valores en la sociedad.

En ambas dimensiones, la bioética supone un desafío para el pensamiento, pues pone en cuestión conceptos y supuestos que han sido objeto de debate filosófico. Así, la naturaleza humana, la dignidad, la responsabilidad, etc. son cuestiones que se replantean con una nueva perspectiva. Particularmente en el terreno de la neurociencia, en donde la preocupación por explicar el modo de conocimiento humano, los comportamientos, la toma de decisiones e incluso los sentimientos, dan lugar a reinterpretar, con una luz diferente, temas como la conciencia, la libertad, la imaginación, los contenidos mentales o la identidad moral de las personas.

Muchos autores hablan de una filosofía naturalizada como característica propia de esta aproximación en la que los datos científicos son tan relevantes. Desde esta perspectiva, deudora de la propuesta de epistemología naturalizada que hiciera W.V. Quine en los años 60 del siglo XX (Quine, 1969), se asume que el modo de revelar cómo podemos conocer la realidad se deriva de una explicación biológica sobre el funcionamiento del cerebro humano (Churchland, 1987). Esto supone asumir varias afirmaciones, de las cuales la primera y más importante es que el cerebro es fruto de la evolución, lo que nos asemeja a otras especies en la medida en que se den capacidades derivadas de desarrollos similares del sistema nervioso. Esa dimensión evolutiva también nos permite comprender que la cognición no puede separarse del "nicho ecológico" que la sustenta, la estructura corporal. Es decir, que lejos de entender el cerebro como una mera computadora capaz de realizar operaciones generales -como quizá en su día defendió la metáfora fuerte del ordenador para explicar el funcionamiento del cerebro-, más bien es preciso observar el cerebro como una herramienta para la supervivencia del individuo. Así, un mayor control sensoriomotor y una mayor velocidad de procesamiento le dan al sujeto más posibilidades de sobrevivir.

Por otra parte, la misma investigación neurocientífica, sus métodos y posibilidades, y las aplicaciones futuras que puedan preverse, como por ejemplo la mejora cerebral, generan importantes interrogantes éticos, algunos de los cuales no distan demasiado de reflexiones y análisis que ya se han elaborado previamente para otras cuestiones similares -por ejemplo, en ética de la investigación con seres humanos-, quedando así emplazados dentro de la tarea bioética en la que se inscribe buena parte del trabajo de la neuroética. Sin embargo, para otros problemas tendremos que arbitrar nuevas estructuras de análisis, puesto que su novedad e implicaciones exceden los territorios ya explorados. Los conceptos y teorías que se están manejando en la investigación neurocientífica, y que condicionan tanto el diseño de los experimentos como la interpretación de los resultados, afectando por tanto a su validez, son cuestionables. Es esencial analizar en profundidad las teorías filosóficas subyacentes. De este modo, la propia neurociencia se convierte en objeto de examen. 
¿Cuál sería la actitud de Xavier Zubiri ante los debates suscitados en la bioética, y específicamente en la neuroética? Sin duda, lo primero que cabe afirmar es que estaría fascinado. Interesado y atento a los descubrimientos científicos, preocupado por su repercusión en conceptos y problemas filosóficos, y madurando con prudencia su propia reflexión. Dado que ciencia y filosofía son para él, aunque diferentes, inseparables, como momentos unitarios de la investigación, los nuevos avances en las ciencias lo arrastrarían a una búsqueda inacabable. En sus propias palabras:

«Nada es real si no es respecto a otras realidades. Lo cual significa que toda cosa real es desde sí misma constitutivamente abierta. Sólo entendida desde otras cosas, que habrá que buscar, habremos entendido lo que es la cosa que queremos comprender. Lo que así entendemos es lo que la cosa es en la realidad. El arrastre con que nos arrastra la realidad hace pues de su intelección un movimiento de búsqueda. Y como esto mismo sucede con aquellas otras cosas desde las que entendemos lo que queremos entender, resulta que al estar arrastrados por la realidad nos encontramos envueltos en un movimiento inacabable. La investigación es inacabable no sólo porque el hombre no puede agotar la riqueza de la realidad, sino que es inacabable radicalmente, a saber, porque la realidad en cuanto tal es desde sí misma constitutivamente abierta.» (Zubiri, 2004, p.7)

\section{EL PROBLEMA DE LA CONCIENCIA Y LA INTELIGENCIA}

El problema metodológico más acuciante al que se enfrentan los estudios sobre la conciencia es el relativo a la conexión o traducción que pueda establecerse entre la descripción de la experiencia subjetiva, que está ligada necesariamente a una perspectiva individual, en primera persona, y los elementos que puedan ser descriptibles objetivamente, mediante los experimentos que adoptan una perspectiva en tercera persona. Así, la cuestión central es si los estados físicos del sistema nervioso humano se "encuentran" en el contenido de la experiencia consciente.

A pesar de que los datos de nuestra percepción son reales -y en ello insistió notablemente Zubiri al hablar de la impresión de realidad en el ser humano-, en buena medida, la descripción fenomenológica que realizamos a partir de esas impresiones es un relato desde la experiencia subjetiva, que no es posible extrapolar o universalizar. Este problema es al que se enfrentan las neurociencias actualmente: cuál es la conexión entre el cuerpo viviente y el cuerpo vivido, entre la experiencia subjetiva y las descripciones ob- jetivas, la duda acerca de si, por ejemplo, la activación cerebral que pueda hacerse patente a través de una técnica de neuroimagen revela cuál es el contenido del estado mental que el sujeto experimenta, y si hay algún tipo de relación causal entre la descripción empírica y la experiencia personal.

En 1996 Francisco Varela proponía una unión de la fenomenología de Husserl y las ciencias cognitivas, que dio en llamar "neurofenomenología" (Varela, 1996), y que ha sido desarrollada por otros autores posteriormente (Varela, 1997; Petitot, Varela, Pachoud y Roy, 1999; Bayne, 2004; Gallagher y Varela, 2001). Varela consideraba que esta aproximación metodológica sería capaz de afrontar el duro problema del "hiato explicativo" (explanatory gap), es decir, el espacio existente entre la experiencia fenoménica y la naturaleza física, o lo que también se ha venido denominando la distancia entre el método de primera persona (más propio de la fenomenología) y el método de tercera persona (propio de las ciencias empíricas). La neurofenomenología vendría a resolver este problema, posibilitando un estudio riguroso de la conciencia.

Sin embargo, construir un puente entre la fenomenología y la neurociencia no es fácil. El método propuesto por Varela es la "restricción recíproca". Se trataría de establecer una serie de principios de conexión, sobre la base de las correlaciones entre los datos fenoménicos y los datos neurocientíficos. Otra posible aproximación es la metodología heurística, de acuerdo a la cual los datos fenomenológicos podrían utilizarse como guía en el descubrimiento de datos neurocientíficos, y viceversa. También hay autores que apuntan a un método de establecimiento de relaciones causales entre lo fenoménico y lo neurocientífico. En todos los casos, queda en duda si realmente se logra cerrar ese hiato, si es posible correlacionar adecuadamente la experiencia subjetiva y los datos empíricos de la neurociencia, de modo que puedan aportar una explicación consistente y universal.

Para aclarar esta cuestión, seguiremos el ejemplo que expone Thomas Metzinger (2000): supongamos que una persona visita un laboratorio experimental de filosofía de la mente, allí tiene la oportunidad de sostener en su mano una porción de un cerebro humano, y tiene la experiencia del color gris y la blandura del fragmento. Por la noche, esa misma persona se despierta de un sueño en el que ha revivido exactamente la misma escena y las mismas sensaciones visuales y táctiles. ¿Cuál es la diferencia entre ambos episodios? En el primero, el estado mental se refiere 
realmente a algo existente en el mundo externo, ya que la persona sostenía efectivamente una porción de cerebro en la mano. En el segundo, no estaba presente ese fragmento de cerebro, la persona estaba durmiendo. Sin embargo, desde el punto de vista del contenido de la experiencia, son exactamente iguales. Más aún, sin algún elemento externo que venga a demostrarle a la persona que se encuentra en situación de vigilia, no hay posibilidad ninguna de que pueda diferenciar ambas experiencias, ni determinar el grado de "realidad" que cada una de ellas tiene-entendiendo por realidad la correlación con el elemento externo que suscita la experiencia-. En la filosofía de Zubiri tiene perfecto sentido afirmar que ambas son reales en la aprehensión, si bien eso que es dado en la aprehensión nos lanza más allá, en busca de la realidad del mundo.

En el fondo, esto es algo de lo que tenemos una inquietante experiencia todas las personas: la ausencia de una completa seguridad acerca de nuestra propia existencia, de nuestros propios pensamientos o de nuestra propia conciencia. Es lo que la exitosa película de Christopher Nolan, Inception (Origen) quiere plantearnos: ¿cómo podríamos saber si estamos soñando, cómo distinguir la realidad de la ficción, cómo generar ideas que cambien la identidad y las acciones de los individuos? No es un tema nuevo en el cine, algo de esto se encontraba en la archiconocida y mítica $\mathrm{Ma}$ trix, o en la menos conocida pero sorprendentemente profunda The Thirteenth Floor.

Esto es lo que planteara Descartes, al sospechar que todo lo que habita la mente puede no tener un correlato empírico. En sus Meditaciones explicaba la dificultad de saber cuál es la "realidad real", pues los sueños lo parecen mientras estamos soñando, pero otro tanto ocurre en la situación de vigilia. Por supuesto, la película de Nolan no se limita a establecer esta duda, sino que también invita al espectador a un mimético desenmascaramiento de su subconsciente, acompañando al protagonista en el proceso psicológico de hacer visible y "real" lo que sustenta y subyace en los sueños, su búsqueda de su identidad y, de nuevo, la duda sobre si ésta es más real en la realidad o en los sueños. De hecho, el poder de la creatividad y el ensalzamiento de los sueños está presente en muchas de las expresiones de los personajes de la película, al destacar su capacidad de generar mundos imaginados, de hacer posible lo imposible, e insistiendo en que la realidad dada ya no es suficiente.

Probablemente esto guarda relación con la convicción zubiriana de que el ser humano tiene una capaci- dad de forjar lo irreal, dada su situación vital de fluencia, de oscilación entre lo real y lo irreal. Los proyectos, según este autor, suponen un grado de irrealidad, que obliga a ir más allá del atenimiento a la realidad, hacia el ámbito de lo irreal. Dicho de otro modo: lo irreal es necesario para poder vivir en la realidad (Zubiri, 2005).

La sospecha sobre la realidad y la incapacidad de distinguirla del sueño también se encuentra en la obra de Unamuno "Niebla" de 1914, donde su propio personaje pone en cuestión si el autor no será él mismo un ser soñado, creyendo en una realidad ficticia. $Y$ afirma: "¿qué es el mundo real sino el sueño que soñamos todos; el sueño común?". Y aparece también en autores más clásicos, pues es lo que comenta Pedro Calderón de la Barca en "La vida es sueño". Estas son las palabras de Segismundo:

«Cielos, si es verdad que sueño, suspendedme la memoria, que no es posible que quepan en un sueño tantas cosas. ¡Válgame Dios! ¡Quién supiera o saber salir de todas, o no pensar en ninguna!.» (Calderón de la Barca, 1647/1997, p. 183)

"Estamos en un mundo tan singular, que el vivir sólo es soñar; y la experiencia me enseña que el hombre que vive sueña lo que es hasta despertar. (...)

Sueña el rico en su riqueza que más cuidados le ofrece; sueña el pobre que padece su miseria y su pobreza; sueña el que a medrar empieza, sueña el que afana y pretende, sueña el que agravia y ofende; y en el mundo, en conclusión, todos sueñan lo que son, aunque ninguno lo entiende.

Yo sueño que estoy aquí destas prisiones cargado, y soñé que en otro estado más lisonjero me ví. ¿Qué es la vida? Un frenesí. ¿Qué es la vida? Una ilusión, una sombra, una ficción, y el mayor bien es pequeño; que toda la vida es sueño, y los sueños, sueños son.» (Calderón de la Barca, 1647/1997, pp. 156-157).

Podemos tener un conocimiento de la realidad externa a nosotros mismos, y también de los contenidos mentales de nuestra experiencia, sin embargo, la situación del ser humano, desde el punto de vista epistemológico, sería la de una limitación intrínseca, que los datos científicos no pueden superar. No hay ninguna característica de nuestros contenidos mentales que marque la diferencia entre vigilia y sueño. El recurso que Inception utiliza del totem (una pequeña pieza u objeto) como dato de realidad, no es posible, pues también puede ser soñado.

Pues bien, siguiendo las distinciones típicas de la fenomenología, podemos afirmar con Metzinger, que el 
contenido fenoménico de las representaciones mentales es independiente de que sea verídico. Es algo que se da en la experiencia, consciente o no, desde una perspectiva en primera persona y, sin duda, está determinado por propiedades internas del cerebro. La posibilidad de una descripción científica objetiva o de unos modelos científicos de la conciencia pasa necesariamente por una cooperación intersubjetiva. Si los individuos humanos tienen la experiencia de un cerebro gris y blando, sus propios cerebros activarán los modelos fenoménicos individuales de la realidad. Desde un punto de vista empírico, todos esos cerebros están implicados en una relación perceptiva sobre datos visuales y táctiles. Los modelos de realidad se anclan en los modelos fenoménicos, lo que a este autor le lleva a pensar que se caracterizan por un cierto realismo ingenuo.

Zubiri se enfrentó al mismo problema, pero su solución es algo diferente. Los juicios de la razón son necesariamente provisionales, históricos, revisables y no pueden considerarse absolutamente verdaderos. Ello es debido a que la riqueza de la realidad es inagotable y a que nuestra capacidad de comprensión y explicación, basada en datos empíricos y juicios de experiencia, es limitada. Sin embargo, la razón esboza hipótesis basándose en las experiencias, en las percepciones, y éstas no son meras construcciones vacías, sino intuiciones primarias de una realidad que se nos impone.

Aquello a lo que la inteligencia tiene acceso es a la realidad, y esto no es una forma de realismo ingenuo. Lo sería si pensáramos que podemos tener un acceso directo a la realidad y, con ello, alcanzar una verdad como adecuación de nuestro entendimiento con la cosa misma. Sin embargo, lo que Zubiri dice es bien diferente: se trata de afirmar que en el acto de intelección, lo que se intelige es real, y que el ser humano tiene la capacidad no sólo de aprehender algo, sino de aprehenderlo como real. Desde aquí se podrán realizar las descripciones de esa experiencia de un modo más o menos afinado, pero siempre estarán basadas en algo real. Y, por supuesto, en esas descripciones se harán patentes los datos de las neurociencias -o de cualquier otra ciencia pertinente a la cuestión-, los presupuestos lingüísticos y metodológicos propios de una determinada altura de los tiempos y, en definitiva, los factores histórico-culturales que influyan en las explicaciones.

Lo radical de la aportación zubiriana es la afirmación de que esa capacidad, específicamente humana, de habérselas con las cosas como realidades es lo que formalmente constituye la inteligencia.
«La inteligencia no está constituida, como viene diciéndose desde Platón y Aristóteles, por la capacidad de ver o de formar «ideas», sino por esta función mucho más modesta y elemental: aprehender las cosas no como puros estímulos, sino como realidades. Toda ulterior actividad intelectiva, es un mero desarrollo de ésta su índole formal.» (Zubiri, 1963, p. 18)

Las cosas pueden presentarse como estímulos y como realidades, pero el ser humano tiene la posibilidad de hacerse cargo de la situación y entender los estímulos como reales. Se convierten entonces los estímulos percibidos en algo que no sólo afecta al organismo, sino que posee una estructura "de suyo". Esto es posible porque el ser humano tiene un cerebro hiperformalizado, más desarrollado y capaz de elaborar diferentes respuestas ante un mismo estímulo, por tanto relativamente indeterminado. Lo cual significa que el ser humano es una realidad constitutivamente abierta.

Se puede decir así que la inteligencia tiene una función biológica: hacerse cargo de la situación para seleccionar la respuesta más adecuada. Desde esta perspectiva, también Zubiri podría afirmar que es pertinente la convicción de la epistemología naturalizada de que el cerebro nos adapta evolutivamente para la supervivencia. Sin embargo, va más allá.

«En primer lugar, su función primariamente biológica. Inteligir es algo irreductible a toda forma de puro sentir. Pero sin embargo, es algo intrínsecamente "uno» con esta última función. Y esto, por lo menos, en tres aspectos : $a$ ), el cerebro no intelige, pero es el órgano que coloca al hombre en la situación de tener que inteligir para poder perdurar biológicamente; el cerebro tiene, en este aspecto, una función exigitiva, precisamente por su hiperformalización; $b$ ), pero el cerebro tiene una función aun más honda en orden a la intelección: es que sin la actividad cerebral, el hombre no podría mantenerse en vilo para inteligir; c) el cerebro no sólo «despierta» al hombre y le «hace tener que» inteligir, es que además, dentro de ciertos límites, perfila y «circunscribe el tipo» de posible intelección. De aquí que, a pesar de que inteligencia y sensibilidad, sean irreductibles, sin embargo constituyen una estructura profundamente unitaria. No hay cesura ninguna en la serie biológica. En el hombre, todo lo biológico es mental, y todo lo mental es biológico.» (Zubiri, 1963, p. 19)

Pero también, al situarlo en la realidad, la inteligencia deja al ser humano en el mundo de las posibilidades, en una situación de apertura al mundo. Desde aquí es 
desde donde se puede justificar que el ser humano ha de hacer su vida, ha de crear su propia historia y de ello es de lo que ha de hacerse responsable. Por eso, necesariamente, el ser humano está abocado a la ética.

En este necesario realizar su vida apropiándose de determinadas posibilidades y no de otras es donde surge la realidad moral.

"La realidad sustantiva cuyo carácter "físico" es tener propiedades necesariamente por apropiación, es justo lo que yo entiendo por realidad moral.» (Zubiri, 1986, p. 345)

Es decir, la sustantividad humana tiene la característica de apropiarse posibilidades de vida, de manera inexorable, de tal modo que "lo" moral es una dimensión "física" del ser humano, podríamos decir que le es inherente, el ser humano es realidad moral porque es sustantividad personal.

"Que el hombre tenga una dimensión moral es algo que pertenece a su realidad "física". La virtud no es ciertamente algo que el hombre tenga por naturaleza, pero es algo más que un mero valor: es una apropiación real y física de determinadas posibilidades de vida, es decir, es un momento de mi ser personal, de mi personalidad. $Y$ justo esto es lo que a mi modo de ver constituye la dimensión moral del hombre, "Io" moral del hombre. No es necesario que el hombre tenga tal virtud determinada, pero es físicamente inexorable que tenga alguna. Lo cual quiere decir que "Io" moral es una dimensión "física" del hombre. Lo moral es a su modo físico. "La" moral en el sentido de valores, bienes, y deberes sólo es posible fundada en "Io" moral del hombre. Sólo hay bien moral porque el hombre es moral.» (Zubiri, 1984a, p. 207)

\section{LOS CORRELATOS NEURALES DE LA MORAL Y EL SER HUMANO COMO SER MORAL}

Otro planteamiento de la investigación neurocientífica, ligado al anterior, que aún va más allá en su intento de explicar la mente o la conciencia, es la búsqueda de sus correlatos neurales. Se trataría de aislar los correlatos neuronales mínimos suficientes para tipos específicos de contenidos fenoménicos. Aunque esto suponga necesariamente que dichos correlatos sean relativos a un sistema, y que estén influidos por condiciones internas y externas, permitiría describir un conjunto mínimo suficiente para activar ciertos contenidos conscientes en la mente del sujeto.

El problema, de nuevo, reside en los posibles malentendidos y reduccionismos, pues es evidente que mostrar empíricamente la existencia de una correlación no es lo mismo que lograr una explicación. Se van definiendo en los diversos estudios correlaciones entre estados o procesos cerebrales y estados de conciencia, pero no queda claro si hay una relación causal entre ellos. De hecho, las interpretaciones podrían ser muy variadas: desde el monismo materialista más extendido que entendería que el estado mental y el estado cerebral son idénticos, son la misma cosa, si bien vista desde el lado del sujeto o desde el dato objetivo; hasta una posición dualista, que considerase el estado mental como algo completamente diferente del proceso cerebral; o un resultado suyo, en la interpretación epifenomenalista. Con ello se pone de manifiesto que el papel de las teorías es esencial para poder dar sentido a los datos obtenidos en la investigación empírica, y que ninguna afirmación o hipótesis está exenta de valores y conceptos propios del paradigma vigente en el que se inscribe.

Con este tipo de respuestas se pretende resolver el clásico problema mente-cerebro. Es un notable ejemplo de cómo todas estas investigaciones están poniendo en cuestión la propia labor de la filosofía (Brook y Akins, 2005), cuya validez y necesidad son puestas en duda por quienes consideran que sus problemas serán resueltos ahora por las neurociencias, en una suerte de renovado positivismo que, sin embargo, tiene una insospechada fuerza. Como dice Zubiri:

«El auge del ciencismo viene determinado no tanto por un racionalismo o por una crítica positivista del conocimiento, como por esta convicción profunda de que en la ciencia se sirve al hombre la única parcela de realidad que le es accesible con certeza.» (Zubiri, 1987, p. 91)

Algunos autores consideran que el exceso de énfasis en los logros de la neurociencia para dar respuesta a los problemas filosóficos da lugar a un reduccionismo simplificador y materialista en las explicaciones o, lo que es peor, a un absurdo que Maxwell Bennet y Daniel Hacker denominan "falacia mereológica" (Bennett y Hacker, 2003; Bennett, Dennett, Hacker y Searle, 2007). Según estos autores, las descripciones neurocientíficas atribuyen toda clase de funciones al cerebro, convirtiéndose éste así en el agente de dichas acciones. Es decir, se le asignan al cerebro poderes y actividades que corresponden a un sujeto sintiente, lo que supone un grave error, al tomar la parte por el todo.

En la misma línea se sitúa Alva Noë al afirmar que «el sujeto que experimenta no es una parte del cuerpo. No somos nuestro cerebro, sino que el cerebro es una 
parte de lo que somos.» (Noë, 2010, p. 24) Y por ello, para entender fenómenos complejos, como la conciencia, la capacidad de pensar, sentir y comprender el mundo, «debemos considerar un sistema más amplio, del que el cerebro no es sino un elemento más. (...) La conciencia requiere la operación conjunta del cerebro, el cuerpo y el mundo.» (Noë, 2010, p. 27) No se trata de que la mente sea sustituida por el cerebro, como parecen defender autores como P. Churchland (1989), y que, por tanto, las explicaciones teóricas puedan suplirse con explicaciones empíricas, sino de que el cerebro es el sustrato material de la mente, pero ésta es propia de los sujetos, no del cerebro por sí mismo. Los cerebros no tienen mente, las personas sí.

Los trabajos de Pedro Laín Entralgo sobre cuerpo y mente son buena muestra de que Zubiri coincidiría en estas afirmaciones, pues están construidos en buena medida sobre la filosofía de este autor (Laín Entralgo, 1989 y 1991). La concepción zubiriana del ser humano como un sistema de notas, de las cuales una es la inteligencia que procede de un "dar de sí" de la materia misma, hace pensar que el cerebro sólo puede considerarse como el órgano que soporta la capacidad de inteligir, pero que es del ser humano, como un todo, del que puede decirse que piensa, razona, siente o decide.

El ser humano es una sustantividad con carácter psico-orgánico, lo cual supone la existencia de dos subsistemas de notas, unas orgánicas y otras psíquicas. Pero el sistema configurado por unas y otras tiene propiedades que corresponden al conjunto, no a ninguna de las notas constitutivas. Como señala Diego Gracia (2009), el psiquismo específicamente humano es el resultado del proceso de complejización de las propias estructuras de su realidad. Es una nueva función, surgida por sistematismo de las propias estructuras biológicas. Pero la inteligencia no podría ser el resultado del mero sistematismo de notas materiales, pues es una cualidad nueva de enorme importancia, que ha de afirmarse con carácter esencial y constitutivo. Por tanto ha de ser una nota elemental, no una nota sistemática.

«No es una nota sistemática. Se trata, por el contrario, de un elemento nuevo pero elemental, bien que exigido desde las estructuras materiales hiperformalizadas e intrínsecamente y formalmente modulado por ellas.» (Zubiri, 1984b, p. 97)

La tesis de Zubiri es emergentista: no es posible saltar del orden de la estimulidad al orden de la realidad, lo cual significa que por mera complejización de las estructuras no puede darse la "elevación" de la materia a in- teligencia, sin embargo dicha complejización es imprescindible para que ese salto se produzca. Posiblemente, los datos de la neurociencia actual habrían permitido a Zubiri articular mejor y en más profundidad estos planteamientos. No obstante, no cabe duda de que sería defensor de una posición más holística y basada en interacciones complejas, que de un reduccionismo materialista como el que ahora resulta más frecuente.

\section{LA IDEA DE UNA NATURALEZA HUMANA}

Los debates filosóficos propiciados por la neurociencia no son nuevos. Como se ha indicado, retoman en buena medida viejas discusiones -y en muchas ocasiones sin el suficiente bagaje como para interpretar adecuadamente los nuevos resultados empíricos- No es extraño, pues, observar cómo los grandes filósofos han abordado estas cuestiones con una sorprendente actualidad. Aunque sus datos sean anteriores al gran avance del conocimiento generado en la llamada década del cerebro -los años 90 del siglo $X X-$, Zubiri está situado en el mismo tipo de debate que en la actualidad sostienen autores como Steven Pinker y otros acerca de la existencia de una naturaleza humana.

Pinker afirma que existen diferencias innatas de comportamiento que resultan significativas entre los individuos, y también entre los varones y las mujeres. Este tipo de planteamientos ha reabierto un viejo debate relativo a la influencia de lo biológico-genético y lo ambiental-cultural en la configuración de los sujetos. Es la clásica distinción entra naturaleza y cultura, que vuelve a resurgir al enfatizarse los aspectos neurofisiológicos del ser humano y considerarlos determinantes.

Según Pinker (Pinker, 1995, 2001 y 2003), el miedo a afirmar una naturaleza humana, con raíz en la genética, responde a cuatro temores básicos: en primer lugar, la desigualdad, ya que si las personas tuvieran, por naturaleza, facultades mentales diferentes, esto significaría una mejor dotación para ciertas actividades, lo cual podría dar lugar a discriminaciones o a una nueva forma de eugenesia. En segundo lugar, la imperfección, pues si la gente tiene ciertos sentimientos de modo innato, todo afán socio-político por crear un mundo más justo es una pérdida de tiempo. El cambio social sólo puede tener lugar si hay espacio para la transformación. En tercer lugar, el determinismo, es decir, la imposibilidad de atribuir responsabilidad a los agentes, pues ellos estarían siempre actuando conforme al patrón que marca su cerebro, sus genes o su evolución. En último lugar, el nihilismo, en tanto que si los motivos 
y los valores humanos son productos de la fisiología cerebral, forzados por la propia evolución, en realidad no existirían, tan sólo serían resultados explicables biológicamente.

En su opinión, todos estos miedos obedecen a concepciones erróneas y no se siguen necesariamente de la afirmación de una naturaleza humana. Por ello, considera que es compatible la afirmación de la libertad, de la toma de decisiones o del perfeccionamiento por medio de factores ambientales, aun asumiendo la existencia de una naturaleza dependiente de factores genéticos. Por ello critica tres afirmaciones frecuentes, que responden a ideas bastante clásicas: en nuestro mundo contemporáneo se habría instalado, según su diagnóstico, una convicción acerca de que la mente es una tabla rasa, de modo que no existen capacidades o temperamentos innatos, sino que todos son generados por el aprendizaje, la cultura y la sociedad. También, al afirmar esta condición, el ser humano sería bueno por naturaleza, el mito del buen salvaje que no tiene maldad inherente, sino generada por una corrupción externa propiciada por la sociedad. Y, finalmente, en la medida en que lo más importante de nosotros mismos es de alguna manera independiente de nuestra biología, nuestras posibilidades de experiencias y decisiones no pueden explicarse por razones fisiológicas ni evolutivas. Estas son las ideas que se ven comprometidas por los datos de las ciencias actuales, especialmente la genética y la neurofisiología. No obstante, la evidencia empírica se interpreta como una amenaza y, por ello, se combate en nombre de la defensa del ser humano.

Pinker no tiene reparos en afirmar, a partir de los datos científicos, que existen diferencias biológicas -genéticas, neurológicas, etc. - entre los individuos, y que éstas son determinantes. En este sentido, los seres humanos no serían tablas rasas, sino que dispondrían de una serie de posibilidades o limitaciones innatas, que, no obstante, pueden ser, al menos parcialmente, modificadas. Enfatizar estos elementos frente a la fuerza de las explicaciones socioculturales no es, en su opinión, incompatible con la defensa de los más vulnerables, con la lucha por la igualdad, o con la búsqueda de la justicia. Por ejemplo, en relación a las diferencias entre varones y mujeres, Pinker subraya la importancia capital que tiene distinguir entre la proposición moral de que las personas no deben ser discriminadas por motivo de sexo, que él considera el núcleo del feminismo, y la proposición empírica de que hombres y mujeres son biológicamente indistinguibles (Feito, 2008). Para él, resulta evidente que son dos cosas diferentes, y que la distinción entre sexos no obsta a la lucha contra la discriminación, sino que, antes bien, considera que es esencial, precisamente para proteger ese núcleo del feminismo (The Science of Gender and Science).

Su compromiso sociopolítico puede ser correcto, pero en el ámbito de la explicación de lo humano se le ha criticado a Pinker por incurrir en ese tipo de reduccionismo que resulta tan frecuente en nuestros días, al pretender que las ciencias serán capaces de ofrecer una explicación completa del ser humano. De hecho, cada vez enfatiza más que es posible explicar la inteligencia humana como un proceso evolutivo de selección natural (Pinker, 2010). Para defender su opinión se apoya en dos hipótesis: por un lado, que los seres humanos evolucionaron para rellenar un "nicho cognitivo", es decir un modo de supervivencia caracterizado por la manipulación del entorno a través del razonamiento causal y la cooperación social. Por otro lado, que las facultades psicológicas que evolucionaron para prosperar en el nicho cognitivo se pueden definir como dominios de proceso de abstracción metafórica y combinación productiva, que se hacen patentes en el lenguaje humano.

Esta cuestión -expuesta aquí de un modo muy resumido-, la idea de una naturaleza humana, está sin duda en la línea de las preocupaciones de Zubiri, si bien su modo de enfocar la cuestión es bien diferente, al referirse a "el hombre" como una suerte de universal abstracto que puede describirse sin atender a los contextos en que vive, a la peculiaridad de su existencia. Cabe preguntarse si es posible realizar tal descripción "pura", sin tomar en consideración, por ejemplo, el factor cultural. Pero es bien sabido que Zubiri no dijo mucho sobre las diferencias entre géneros o sobre el multiculturalismo, lo cual no deja de ser sorprendente.

El ser humano parece irreductible a su naturaleza, pero sin duda es naturaleza, pues su condición física y biológica son inexcusables y condicionan o posibilitan su mismo modo de ser. Pero al mismo tiempo, algo de razón tenía Ortega y Gasset al afirmar que el ser humano tiene historia y que, por tanto, es algo más o diferente, propio de esa formalidad de realidad que coloca a las personas en un espacio construido, propio, habitado y posibilitante que es el mundo.

\footnotetext{
"El hombre es la única cosa que no se comprende adecuadamente por su sola realidad efectiva, sino más bien por sus posibilidades de ser". (Zubiri, 2002, p. 295)

El ser humano, según Zubiri, se encuentra en una situación radicalmente distinta con respecto al resto de los seres vivos, puesto que se encuentra arraiga-
} 
do en su naturaleza y conjugándola con su libertad. La implantación en la realidad forma una unión indisoluble con la libertad que el ser humano va haciendo, por apropiación, conformando de este modo su vida. Por eso el enfrentamiento con las cosas como realidades es específico de una inteligencia sentiente y es también lo que torna la tendencia en volición, y la afección en sentimiento.

Además, como animal de realidades, el ser humano tiene que realizar el modo de realidad que le es propio: el ser persona. Esa peculiaridad es la que le permite que su modo de ser real sea la autoposesión, es decir, estar sobre sí, ser suyo, y esto es lo que le confiere su personeidad, su ser persona. La forma de realidad humana, la personeidad, adquiere después modulaciones concretas que son su personalidad. Cuando hablamos de persona, por tanto, entendemos por tal la unidad concreta de la personeidad según la personalidad. Esto quiere decir que hay un cierto elemento "constructivo" en la configuración de la personalidad, dependiente en parte de las posibilidades biológicas que ese sujeto concreto tiene, y en parte de las posibilidades ambientales, temporales, históricas y culturales que se le abren en su mundo.

“Por ello, la cuestión del ser del hombre jamás podrá ser resuelta por vía naturalista, porque el hombre existe personalmente". En suma, el hombre tiene naturaleza e historia; y en el marco de su naturaleza, el hombre inscribe su existencia personal. De manera que una persona determinada es lo que en su historia y en su vida le pertenece; porque "no todo lo que pasa en mí o conmigo es mío". (Zubiri, 2002, pp. 297-298).

Puede interpretarse que la dimensión de personalidad abre ese espacio histórico y cultural, sin el cual la vida humana no sería realmente humana. El ser humano es agente y autor de su propia vida, constructor de su biografía. Y esa vida que la persona ejecuta también está condicionada por una determinada época, un determinado contexto socio-cultural, unas determinadas peculiaridades individuales, etc., lo que conforma todo un contexto que ya le viene dado a la hora de ejecutar sus acciones.

«El hombre es así, ante todo, agente de sus actos, o lo que es lo mismo, es el agente de su vida: se posee a sí mismo por la actuación de sus potencias y facultades (...) a la vez que agente de su vida, es actor de su propia vida. La persona es en cierto modo, el gran personaje de su vida.» (Zubiri, 1984a, p. 77)

El ser humano se encuentra en un espacio de posibilidades, abierto a la realidad misma por la inteligen- cia. Esta capacidad es la que permite que las acciones ejecutadas lo sean en vista a la propia realidad del sujeto en tanto que realidad. Es decir, el ser humano se "va haciendo" en su fluir en la realidad. Y las acciones realizadas van configurando una figura del yo. La conciencia así no es tan sólo una transición entre estados de cosas, como si sólo fuera un espectador de lo que ocurre, sino que proyecta la propia realidad. El recorrido de la vida humana alcanza su unidad interna completándose y configurándose en la realidad, pues cada acción revierte sobre la realidad del mismo ser humano. (Zubiri, 2005) Por eso Zubiri, al enfatizar este aspecto constructivo, fluyente e inacabado, se apoya en la realidad física y en el ámbito o campo de posibilidades dadas, pero explica al ser humano como una interacción que deviene, cambia, se constituye y da como resultado una nueva realidad.

Probablemente, considerar que el ser humano puede describirse por su naturaleza, es decir por su condición genética, por sus rasgos biológicos -lo que configura su personeidad-, y por su apropiación de posibilidades, la forma de personalidad que va adquiriendo, que es dinámica, abierta, cambiante, e inconclusa, es una visión compleja y acorde con los datos científicos. De esta manera, los avances en la neurociencia actual le proporcionarían a Zubiri nuevas bases en las que sustentar su descripción de la naturaleza humana, pero su planteamiento podría recibir críticas similares a las vertidas contra Pinker, pues enfatizar el arraigo biológico del ser humano es precisamente lo que hace este autor.

Le salva a Zubiri el no haber prescindido de la situación del ser humano como realidad que tiene un mundo, como modo propio de ser. Sin embargo, a la altura de nuestro tiempo, parecería necesario quizá ampliar la descripción, para dar cabida al impacto de lo cultural como auténtico elemento configurador de la identidad. Que los aprendizajes recibidos son capaces de modificar físicamente nuestro cerebro -generando, por ejemplo, nuevas conexiones neuronales - es algo ahora bien sabido, que sin duda llevaría a afirmar la interacción entre lo biológico y lo cultural. Que existen diferencias cerebrales correlativas a las diferencias de costumbres, de género, de lenguaje, etc. también permitiría matizar algunas cuestiones. Que nuestras capacidades tecnológicas tienden a permitir la modificación cerebral y con ello lograr efectos como la mejora de la memoria o la atención, supone también un ámbito de reflexión que obligaría a revisar y completar buena parte de las afirmaciones pues, si los recuerdos forman parte de la identidad de 
la persona, al quedar como el sustrato de las acciones pasadas y las experiencias vividas, poder intervenir en la memoria, además de una esperanza para los enfermos de Alzheimer, puede constituir una inquietante forma de reconfiguración de la personalidad sobre la que es preciso reflexionar.

\section{EL COMPROMISO DEL FILÓSOFO CON LA REALIDAD}

En cualquier caso, el talante zubiriano apuntaría a un intento de articulación entre saberes, a una filosofía a la altura de los tiempos, que pudiera dar cuenta de los datos científicos. Y en esto estaría de acuerdo con Paul Ricoeur:

«No creo exagerado decir que la distancia semántica es tan grande entre las ciencias cognitivas y la filosofía como entre las ciencias neuronales y la filosofía. Esa distancia entre vivencia fenomenológica y dato objetivo recorre toda la línea de división entre las dos aproximaciones al fenómeno humano. Pero ese dualismo semántico (...) no puede ser más que una posición de partida. La experiencia múltiple, amplia y completa está compuesta de tal modo que ambos discursos no dejan de ser correlativos en numerosos puntos de intersección.» (Changeux y Ricoeur, 1999, p. 32)

Y esto es absolutamente necesario, no sólo desde el punto de vista del conocimiento, sino también desde la responsabilidad que tiene el ser humano, en tanto que ser moral. La realidad exige respuestas. El ámbito del conocimiento no puede reducirse a la mera explicación, sino que requiere también la acción, la toma de decisiones, la resolución de los conflictos, la ordenación de la convivencia, y la promoción de ciertos valores. Ésta es la tarea de la bioética en su dimensión aplicada.

En el caso de la neuroética, la búsqueda de los correlatos neurales de la conciencia y la moral puede dar como resultado la afirmación, por ejemplo, de la existencia de ciertos patrones generales de activación cerebral ante ciertos dilemas morales. También una visión simplificadora de los datos de estas investigaciones puede promover un cambio en nuestro concepto de responsabilidad, pues si se establece una relación causal determinista entre un proceso cerebral y un estado mental, podría afirmarse que un sujeto no es responsable, moral o legalmente, de una acción, pues tan sólo obedece necesaria e involuntariamente a su naturaleza. Y quizá, con las nuevas técnicas disponibles, podría proponerse una mejora cerebral de los individuos dotados de ciertas configuraciones cerebrales consideradas menos óptimas para la convivencia. Siendo todo esto posible, es preciso analizar qué consecuencias tendrían estos planteamientos desde un punto de vista social, político y cultural, cómo pueden transformar nuestra cultura y valores, y qué tipo de decisiones conviene adoptar, tanto en la búsqueda de ciertos fines, como en los medios para lograrlos.

Aunque Metzinger opine, como probablemente lo haría el propio Zubiri, que esta otra dimensión de la neuroética es la "blanda", no por ello menos importante, pero sí menos radical desde el punto de vista filosófico, es posible pensar que la metafísica de la realidad de Zubiri, bien podría haber sido, además, una metafísica de la acción. Y quizá, enfrentado a los retos del presente, hubiera desarrollado también esa otra dimensión. De hecho, el propio Zubiri hace patente esa radical determinación física del ser humano como ser moral. Y esto exige otro planteamiento.

La metafísica no tiene que estar necesariamente reñida con la ética. Como dice Reiner Wiehl:

«El conocimiento metafísico es, como cualquier otro conocimiento, parte de este mundo, y por ello se encuentra vinculado necesariamente a la experiencia. Su misión frente a cualquier experiencia dada es, al menos, doble: debe, por un lado, hacerla más visible y transparente; por otro, debe abrir nuevos horizontes para la multiplicidad de experiencias dadas. Las categorías metafísicas son válidas tanto para las experiencias de los mundos de vida multiculturales como para las múltiples experiencias de unas ciencias cada vez más diferenciadas.» (Wiehl, 1992, p. 109)

A la altura de nuestro tiempo, la labor del filósofo no puede ser, exclusivamente, la del pensador alejado de la vida cotidiana, que teoriza sobre el mundo y la realidad, sin meterse de lleno en ella y sus conflictos. Si la bioética tiene algún interés para el filósofo, es precisamente el de retarle a pensar y encontrar soluciones, el de ser capaz de moverse en el terreno de los conceptos y los fundamentos, pero también de aterrizar en la cruda realidad y sus problemas. Esto no implica renunciar a la metafísica, antes bien, exige una metafísica que, además, pueda hablar de la ética, y articular el conocimiento de la realidad en sus múltiples dimensiones. Pues al final, sueño o realidad, como decía Calderón, obrar bien es lo que importa:

«No me despiertes si duermo; y si es verdad, no me duermas. Mas sea verdad o sueño, obrar bien es lo que importa. Si fuere verdad, por serlo; si no, por ganar amigos para cuando despertemos.» (Calderón de la Barca, 1647/1997, pp. 166-167). 


\section{BIBLIOGRAFÍA}

Bayne, T. (2004). Closing the gap? Some questions for neurophenomenology. Phenomenology and the Cognitive Sciences, 3-4, pp. 349364. http://dx.doi.org/10.1023/ B:PHEN.0000048934.34397.ca

Bennett, M., Dennett, D., Hacker, P. y Searle, J. (2007). Neuroscience and Philosophy: Brain, Mind, and Language. New York: Columbia University Press.

Bennett, M. R. y Hacker, P. M. S. (2003). Philosophical Foundations of Neuroscience. Wiley-Blackwell.

Brook, A. y Akins, K. (eds.) (2005). Cognition and the Brain: The Philosophy and Neuroscience Movement. Cambridge University Press. http://dx.doi. org/10.1017/cbo9780511610608

Calderón de la Barca, P. (1647/1997) La vida es sueño. Madrid: Espasa-Calpe.

Changeux, J.-P. y Ricoeur, P. (1999). Lo que nos hace pensar. La naturaleza y la regla. Barcelona: Península.

Churchland, P. (1987) Epistemology in the age of neuroscience. The Journal of Philosophy, 84, 10, pp. 544-553. http:// dx.doi.org/10.5840/jphil1987841026

Churchland, P. (1989). Neurophilosophy: toward a unified science of the mindbrain. Cambridge, MA: MIT Press.

Feito, L. (2008). Cerebros de mujeres y cerebros de hombres ¿Conflicto de racionalidades? En: Feito, L. (ed.) El conflicto de racionalidades. Madrid: Universidad Pontificia de Comillas, pp. 205-215.

Gallagher, S. y Varela, F. (2001). Redrawing the map and resetting the time: phenomenology and the cognitive sciences. En: Crowell, S., Embree, L. y Julian, S. J. (eds.) The reach of reflection: issues for the phenomenology's second century. Center for Advanced Research in Phenomenology.
Gracia, D. (2009). Zubiri en los retos actuales de la antropología. Cuadernos Salmantinos de Filosofía, 36, pp. 103-152.

Laín Entralgo, P. (1989). El cuerpo humano. Teoría actual. Madrid: Espasa Calpe.

Laín Entralgo, P. (1991). Cuerpo y alma. Madrid: Espasa Calpe.

Metzinger, T. (2000). Introduction: Consciousness research at the end of the twentieth century. En: Metzinger, T. (ed.) Neural correlates of consciousness. Empirical and conceptual questions. Cambridge, MA: MIT Press, pp. 1-16.

Noë, A. (2010). Fuera de la cabeza. Por qué no somos el cerebro y otras lecciones de la biología de la conciencia. Barcelona: Kairós.

Petitot, J., Varela, F., Pachoud, B. y Roy, J. M. (eds.) (1999). Naturalizing Phenomenology. Stanford, CA: Stanford University Press.

Pinker, S. (1995). El instinto del lenguaje: cómo crea el lenguaje la mente. Madrid: Alianza.

Pinker, S. (2001). Cómo funciona la mente. Madrid: Destino.

Pinker, S. (2003). La tabla rasa. La negación moderna de la naturaleza humana. Barcelona: Paidós.

Pinker, S. (2010). The cognitive niche: Coevolution of intelligence, sociality, and language. Proceedings of the Natural Academy of Sciences. [On line]. http:// dx.doi.org/10.1073/pnas.0914630107

Quine, W. v. O. (1969). Epistemology naturalized. En: Quine, W. v. O. Ontological Relativity and other Essays. Nueva York: Columbia University Press.

Varela (1996) Neurophenomenology: a methodological remedy for the hard problem. Journal of Consciousness Studies, 3-4, pp. 330-349.
Varela (1997). The naturalization of phenomenology as the transcendence of nature: searching for generative mutual constraints. Alter: Revue de Phénoménologie, 5, pp. 355-385.

Wiehl, R. (1992). Hermenéutica, Metafísica, Ética. La filosofía en lengua alemana durante las últimas décadas del siglo XX. Isegoría, 15, pp. 93-118. http://dx.doi. org/10.3989/isegoria.1992.i5.340

Zubiri, X. (1963). El hombre, realidad personal. Revista de Occidente, 1, pp. 5-29.

Zubiri, X. (1984a). El hombre y Dios. Madrid: Alianza.

Zubiri, X. (1984b). Inteligencia y Realidad. Madrid: Alianza.

Zubiri, X. (1986). Sobre el hombre. Madrid: Alianza.

Zubiri, X. (1987). Naturaleza, Historia, Dios. Madrid: Alianza.

Zubiri, X. (2002). Sobre el problema de la filosofía y otros escritos (1932-1944). Madrid: Alianza.

Zubiri, X. (2004). Sentido de la vida intelectual. En: Nicolás, J. A. y Barroso, O. (eds.) Balance y perspectivas de la filosofía de X. Zubiri. Granada: Comares.

Zubiri, X. (2005). El hombre: lo real y lo irreal. Madrid: Alianza / Fundación Xavier Zubiri.

\section{Recursos de Internet}

The Science of Gender and Science. Pinker vs. Spelke. A debate. [On line]. Recuperado de https://www.edge.org/event/ the-science-of-gender-and-sciencepinker-vs-spelke-a-debate. 